\title{
EDGE DETECTION IN MULTISPECTRAL IMAGES USING THE N-DIMENSIONAL SELF-ORGANIZING MAP
}

\author{
Johannes Jordan, Elli Angelopoulou \\ Pattern Recognition Lab, University of Erlangen-Nuremberg, Germany \\ johannes.jordan@cs.fau.de, elli.angelopoulou@cs.fau.de
}

\begin{abstract}
We propose a new method for performing edge detection in multispectral images based on the self-organizing map (SOM) concept. Previously, 1-dimensional or 2-dimensional SOMs were trained to provide a linear mapping of high-dimensional multispectral vectors. Then, edge detection was applied on that mapping. However, the 1-dimensional SOM may not converge on a suitable global order for images with rich content. Likewise, the 2-dimensional SOM introduces false edges due to linearization artifacts. Our method feeds the edge detector without linearization. Instead, it exploits directly the distances of SOM neurons. This avoids the aforementioned drawbacks and is more general, as a SOM of arbitrary dimensionality can be used. We show that our method achieves significantly better edge detection results than previous work on a high-resolution multispectral image database.
\end{abstract}

Index Terms - Multispectral imaging, Image edge detection, Self organizing feature maps, Machine Vision

\section{INTRODUCTION}

Edge detection is a well-understood preprocessing step for computer vision applications. The Canny operator dates back to 1986 [1], but is still widely in use. However, common methods are limited to monochromatic images. Adapting them to multispectral or hyperspectral images, where each pixel holds a high-dimensional spectral vector is a difficult problem. Previous approaches are either based on the image gradient [2] or on pixel ordering [3]. Gradient-based methods may fail to detect edges in the case of opposing gradients present in different spectral bands $[4,3]$. To avoid this problem, orderingbased methods determine edge probability by a local R-ordering of adjacent pixels. However, it has been shown that like gradient-based methods, R-ordering edge detection is also prone to missing edges [5]. Due to the locality of the ordering, pixels holding different values may be mapped to the same scalar.

In this paper, we take a closer look at the method by Toivanen et al. [5]. It employs a Self-Organizing Map (SOM) [6] to generate a global ordering of spectral vectors. With a global ordering, a one-to-one correspondence between pixel values and scalars is guaranteed. The edge probability is only determined by the adjacent pixels, invariant to regional characteristics.

While this method solves problems present in previous approaches, it is highly dependent on a good global ordering. As such an ordering is hard, if not impossible to find for complex scenes, it suffers from edge artifacts. We discuss this observation in detail and provide a solution to the problem. We show that our variant performs better on images from the high-quality CAVE multispectral image database [7], which is of higher resolution than previously tested images.

\section{SELF-ORGANIZING MAPS FOR EDGE DETECTION}

Toivanen et al. introduced the Self-Organizing Map to the application of edge detection, which is a concept formulated first by Kohonen [6]. This artificial neural network is trained using unsupervised learning to convert the nonlinear statistical relationship between highdimensional data into simpler geometric relationships. In other words, observed spectra are put into a topological relation that is exploitable for data ordering.

We define the data of a multispectral image in the form $\mathbf{v}_{x, y} \in$ $\mathbb{R}^{d}$, whereas $d$ is the number of spectral bands present in the input image and $\mathbf{v}_{x, y}$ corresponds to an image pixel at position $x, y$. The SOM consists of $n$ model vectors $\mathbf{m}_{i} \in \mathbb{R}^{d}$ (also called neurons). Let $d\left(\mathbf{v}_{x, y}, \mathbf{m}_{i}\right)$ denote a distance function for $\mathbf{v}_{x, y}$ and $\mathbf{m}_{i}$. The best fit of $\mathbf{v}_{x, y}$ in the SOM, and therefore its match $\mathbf{m}_{c}$, has the index

$$
c_{x, y}=\underset{i}{\operatorname{argmin}} d\left(\mathbf{v}_{x, y}, \mathbf{m}_{i}\right) .
$$

We use the Euclidean distance for $d(\cdot)$. During training, $s$ vectors from the input image (or any other source) are randomly selected and fed into the SOM. After the match of input $\mathbf{v}_{x(t), y(t)}(1 \leq t \leq s)$ is determined, a neighborhood function $h_{c i}$ defines the influence of $\mathbf{v}_{x(t), y(t)}$ on the model vectors $\mathbf{m}_{i}$. As suggested in the literature [6], we use

$$
h_{c i}(t)=\alpha(t) \cdot \exp \left(-\frac{\left\|\mathbf{r}_{c}-\mathbf{r}_{i}\right\|^{2}}{2 \sigma^{2}(t)}\right)
$$

where $\mathbf{r}_{c}, \mathbf{r}_{i}$ are the location vectors of neurons $c$ and $i$, respectively. The location $\mathbf{r}_{c}$ of a neuron $\mathbf{m}_{c}$ in the SOM is determined by the SOM topology and is a bijective mapping of $c$. The learning-rate factor $\alpha(t)$ is a user-adjustable parameter that is monotonically decreasing. The kernel width $\sigma(t)$ describes how far the influence of a sample vector reaches in the SOM topology and is also monotonically decreasing. While in the early training phase the SOM should seek a rough global ordering, in the later phase local regions are smoothed out. In our experiments, $\alpha(1)=0.1, \alpha(s)=0.001$ and $\sigma(1)=\frac{n}{4}, \sigma(s)=0$.

Toivanen et al. used location scalars $r_{i} \in \mathbb{N}$ or vectors $\mathbf{r}_{i} \in$ $\mathbb{N}^{2}$, resulting in a 1-dimensional SOM (2-connected topology) or 2-dimensional SOM (4-connected topology), respectively. While the former provides a natural way of obtaining a linear order, the latter is better suited for larger SOMs that strive to cover more the complex relationships often found in the input data.

\subsection{The 1-dimensional case}

The order of spectral vectors can be easily generated if neurons of a SOM with size $n$ are ordered by a scalar location index $1 \leq r_{i} \leq n$. We obtain a monochromatic image for edge detection as follows. For each pixel $\mathbf{v}_{x, y}$ of the input image, we determine its match $\mathbf{m}_{c}$. The 


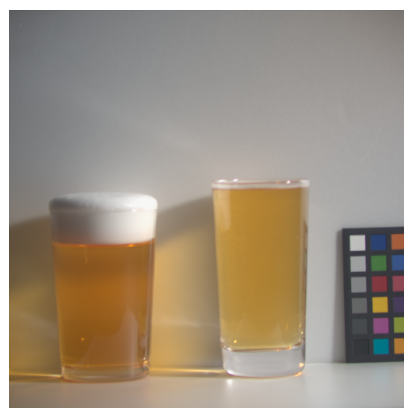

(a) Original image in sRGB

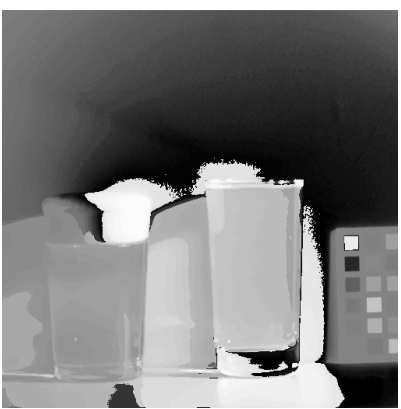

(b) Rank image from 1D SOM
Fig. 1. Example multispectral image illustrating the weakness of 1DSOM. The rank image has outliers dominating other rank transitions; the strongest edges to be detected are false.

location $r_{c}$ is directly used as an intensity value in the rank image $\mathbf{R}$. The edge detector works on $\mathbf{R}$ which is expected to provide a strong gradient between pixels of significantly different spectral responses.

The quality of the obtained ordering depends on the SOM organization. In a well-organized SOM you see smooth transitions between spectra of adjoining neurons as well as a good global clustering of similar spectra. This means that slightly different shades of the same material observed in the scene are expected to be represented by neurons close to each other. In the 1-dimensional SOM, this is violated for images depicting more complex scenes. Each model vector can only be part of two neighborhoods that express a specific proximity relationship in the input data space. For more complex clusters of data in the input space, some proximity relationships are distorted or lost; vectors from one cluster will end up in several distinct locations of the SOM. This can result in edges introduced between pixels that are part of such a cluster. Figure 1 shows an example image from the CAVE database [7] and the rank image obtained by a 1-dimensional SOM. The SOM with $n=64$ neurons was trained with 100000 random samples from the image. It does not relate well the different reflectance effects in the scene.

Due to this aforementioned limitation, the 1-dimensional SOM is not advisable in a larger scale regarding its number of neurons $n$. On the other hand, SOMs of larger $n$ are trained to cover variation in the spectra in more detail and enable a more fine-grained edge detection. A better coverage of detail changes in the image is traded with higher risk of false edges based on amplified location discrepancy.

\subsection{The 2-dimensional case}

The problem of the 1-dimensional SOM described in the previous section may be the reason why SOMs are typically applied with a 2dimensional, 4-connected topology. For spectral vector ordering, this introduces the need for a linearization on the 2-dimensional lattice. Toivanen et al. employ space filling curves [8]. The Hilbert curve and Peano curve describe a recursive rule for traversing a 2-dimensional lattice of size $2^{a} \times 2^{a}$, and $3^{a} \times 3^{a}$, respectively $(a \in \mathbb{N})$.

These curves are designed to provide a close linear index for each pair of 2-dimensional coordinates which would also be close according to their Euclidean distance. The drawback of this method lies in the portion of coordinates where this relationship analogy to the Euclidean distance is violated. Due to the recursive nature of the space filling curve, neurons that are adjacent in the SOM can end up with a difference in the linear index of over $0.8 n$. Therefore, the topological organization of the SOM is not well covered by the resulting order and associated intensity values that are used for edge

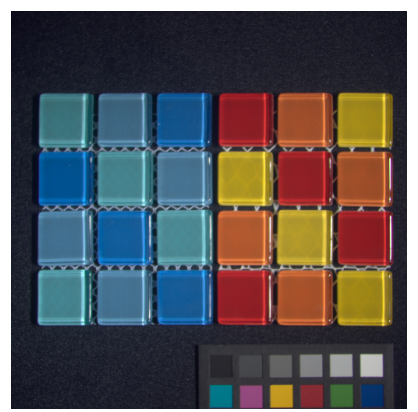

(a) Original image in sRGB

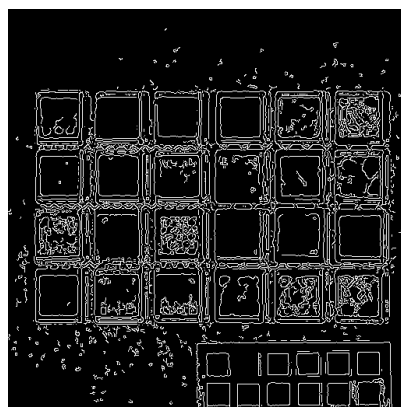

(c) Canny result, orig. 2D SOM

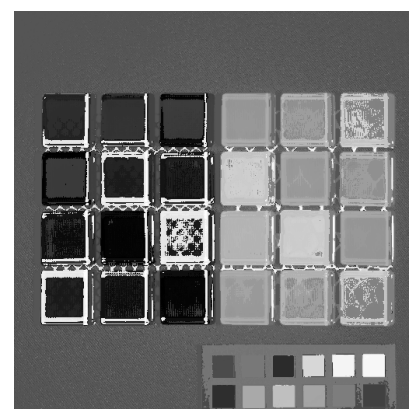

(b) Rank image from orig. 2D SOM

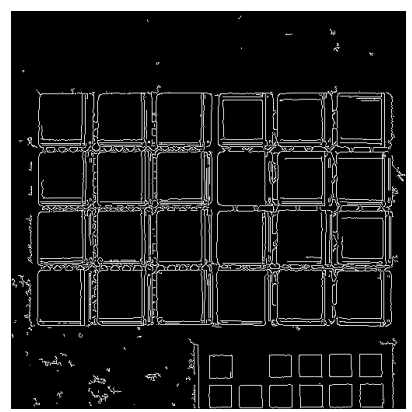

(d) Canny result, new method
Fig. 2. Example multispectral image illustrating the weakness of 2D-SOM. The rank image shows inconsistent ranks for similar pixels. As a result, false edge artifacts can not be avoided without loosing valuable edge information. The new method shows a very consistent edge detection.

detection. This problem is significant, as can be seen in Figure 2 . Depicted results were obtained with parameters set as described in Section 4 (p. 3). Again, strong edges are obtained between pixels of similar spectra. The edge detector cannot prevent false edges without loosing valuable edge information.

\section{NEW METHOD}

The SOM is a useful tool for reducing the high dimensionality of a multispectral image in a data-driven fashion. It provides a lowdimensional topological representation of the contained spectra and their relationship. To make it more usable for the task of edge detection, we must overcome the need of linearization. In fact, the ordering of spectral vectors is not needed for edge detection. To gain differential information that is input to the Canny operator, we can directly exploit the topological information of the SOM.

We create differential maps for the $\mathrm{x}$-direction and the y-direction which replace the first derivatives as input to Canny. To make the Canny operator work as expected, we mimic the $3 \times 3$ Sobel operator. First, each pixel $\mathbf{v}_{x, y}$ in the image is assigned to its match $\mathbf{m}_{c}$ according to Equation 1, resulting in the index map C. Then, we create the differential maps $\mathbf{G}^{(H)}$ and $\mathbf{G}^{(V)}$ using

$$
\begin{aligned}
& g_{x, y}^{(H)}=\| \frac{\mathbf{r}_{c_{x-1, y-1}}+2 \mathbf{r}_{c_{x-1, y}}+\mathbf{r}_{c_{x-1, y+1}}}{4} \\
& -\frac{\mathbf{r}_{c_{x+1, y-1}}+2 \mathbf{r}_{c_{x+1, y}}+\mathbf{r}_{c_{x+1, y+1}}}{4} \|, \\
& g_{x, y}^{(V)}=\| \frac{\mathbf{r}_{c_{x-1, y-1}}+2 \mathbf{r}_{c_{x, y-1}}+\mathbf{r}_{c_{x+1, y-1}}}{4} \\
& -\frac{\mathbf{r}_{c_{x-1, y+1}}+2 \mathbf{r}_{c_{x, y+1}}+\mathbf{r}_{c_{x+1, y+1}}}{4} \|,
\end{aligned}
$$




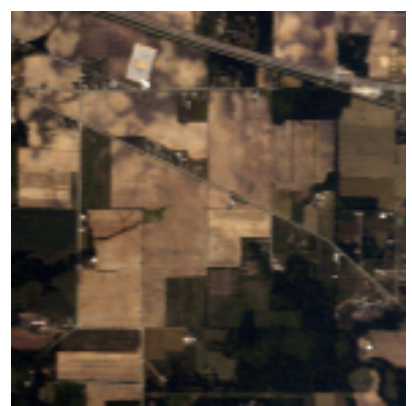

(a) Original image (pseudo-color)

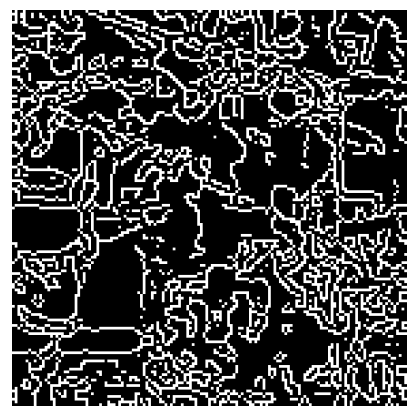

(c) Orig. method, 16x16 SOM

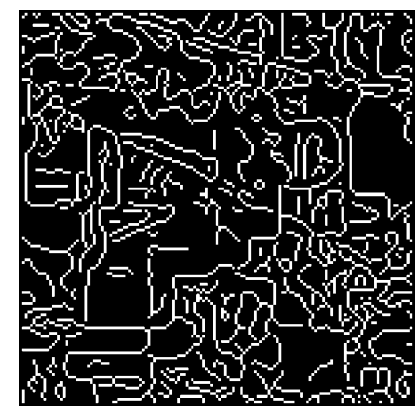

(b) Toivanen et. al, $64 \times 1 \mathrm{SOM}$

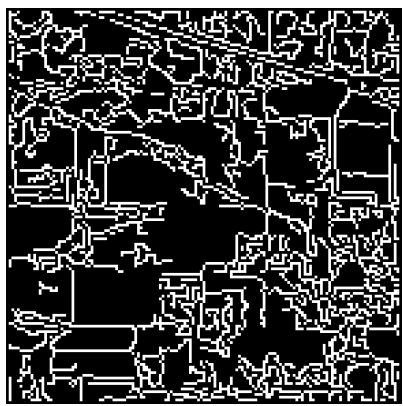

(d) Our method, 16x16 SOM
Fig. 3. Aviris image used for evaluation in [5]. (b) is taken from the original publication, (c) shows the result from our implementation of the original method, (d) our new method using the same parameters.

where $\|\cdot\|$ is the norm used in the neighborhood function (see Equation 2).

In the case of a 1-dimensional topology, this yields the same behavior as before. With a 2-dimensional topology, linearization is omitted and the topological relationship of neurons is the sole origin of $\mathbf{G}^{(H)}, \mathbf{G}^{(V)}$. Besides avoiding artifacts introduced by a linearization, this approach bears two significant advantages that make it a generalization of the previous approaches. First, in the 2-dimensional case we can lift the restrictions on SOM size that were imposed by the space filling curves. SOM size can now be arbitrary instead of being bound to a power of 4 or 9 . Second, the SOM topology can be of higher dimensionality. We deem it reasonable to use a 3-dimensional, 6-connected topology for larger-sized SOMs.

\section{EVALUATION}

A rigorous evaluation of the SOM methodology for multispectral edge detection is lacking in current literature. Toivanen et al. showed results on two remote sensing images of a spatial resolution of nearly $150 \times 150$ pixels [5]. One of which is hyperspectral (220 bands in the range $400 \mathrm{~nm}-2500 \mathrm{~nm}$ ) and of known source [9]. While this image shows the practicability of the method for hyperspectral data, images of higher resolution and a better controlled scene might help to get further insights when comparing different variants of the method. To test our method on the image, we trained SOMs with $n=256(16 \times 16), s=200000$. In our experiments, the training input is always randomly sampled from the same image without further pre-processing. Figure 3 shows how our method performs compared to the result published in [5]. One can observe that our method helps to better discern the edges present in the image, most

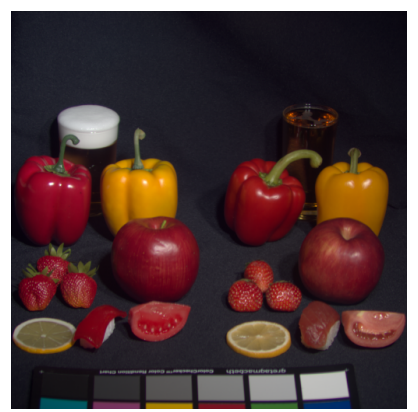

(a) Original image in sRGB

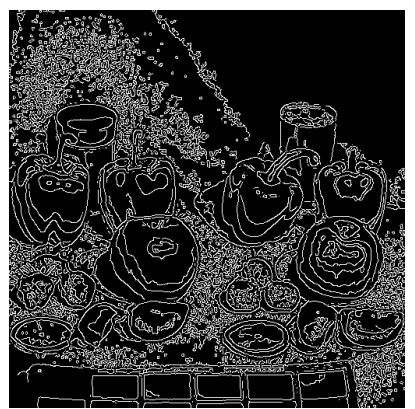

(c) Orig. method, 16x16 SOM; A

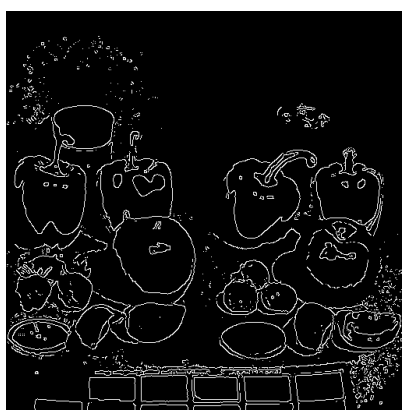

(e) Orig. method, 16x16 SOM; B

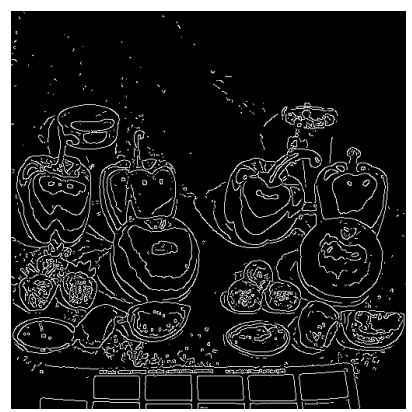

(b) $256 \times 1 \mathrm{SOM}$

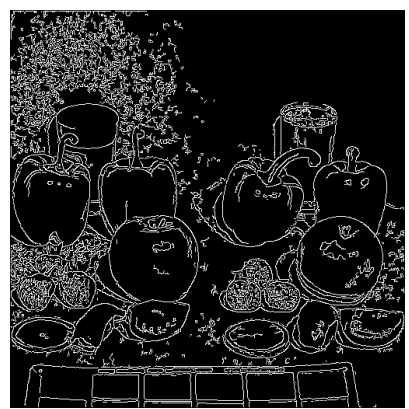

(d) Our method, 16x16 SOM; A
Fig. 4. Edge detection results. Canny parameter criteria: A) best object contour preservation; B) minimum fine-grained noise introduced by object/background texture.

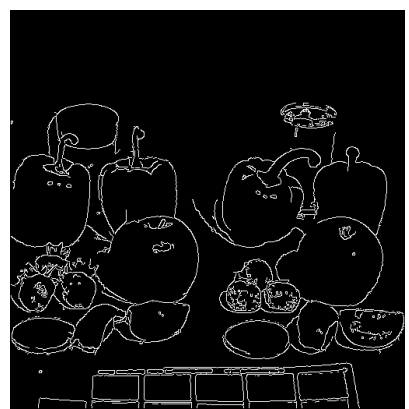

(f) Our method, 16x16 SOM; B

visible at the track on the top. Also, the rectangular structures are better reflected.

\subsection{Images from CAVE database}

We compared our proposed method with previous work on the CAVE multispectral image database. This is a set of high-quality multispectral images that depict objects of different materials in a laboratory setting. Images have a spatial resolution of $512 \times 512$ pixels and cover the spectral range of $400 \mathrm{~nm}-700 \mathrm{~nm}$ in 31 bands. We trained SOMs with the same parameters as before.

Figure 4 shows results on an image depicting fake and real food items. We observe that the 1-dimensional SOM provides a clear picture, but edges detected due to geometry effects distract from the object boundaries. For the 2 -dimensional SOMs $(16 \times 16)$, we depict edge images created with Canny hysteresis parameters chosen for two different criteria. We observe that due to linearization artifacts, the original method introduces many edges on originally smooth shade 
changes. Based on Canny parametrization, object contours are either not well-distinguishable or missed in the edge image. Contrary, the new method embosses object contours as well as specular highlights, enabling a good interpretation for scene understanding.

Figure 5 shows results on an image depicting an Egyptian statue next to a stuffed toy. As can be seen in the second row, the SOM manages to preserve structure that is in shadow and only perceptible in a small amount of spectral bands (marked in red). With the 1dimensional SOM, the contour of the shadowed throat is lost while smooth transitions in the face still lead to edges. A similar effect is visible with the 2-dimensional SOM using a space filling curve. Contrary, the new method manages to expose characteristics of face, hat and toy while maintaining a low noise level.

\subsection{Discussion}

Toivanen et al. found that on their two remote sensing images, a 1-dimensional SOM and a 2-dimensional SOM both give comparable results [5]. However, given the image size, they can only observe a considerably small amount of different spectral vectors. When testing on newer images, we have a significantly higher variety of the data which may not be well-represented by a small-sized SOM. We observe that the 1-dimensional SOM performs worse and the original 2-dimensional SOM suffers from linearization artifacts. As a result, our proposed method achieves better edge detection on the tested images. The quality we obtain shows that SOMs are a useful tool for multispectral edge detection also on images with higher resolution.

\section{CONCLUSIONS}

Edge detection on multispectral and hyperspectral images is an issue that has not yet been sufficiently addressed. Previous work on R-ordering was an important step in better reflecting the highdimensional characteristics of the data when compared to gradientbased methods. With the Self-Organizing Map we have a promising methodology at hand that already provides usable results. However, the method as previously presented suffered from its ties to pixel ordering. Our method omits linearization and uses the SOM more efficiently for edge detection while also retaining greater flexibility. Our experiments demonstrate that artifacts introduced by linearization can be avoided without a disadvantage in algorithm performance. Good edge maps can be found on images that reflect the current state of multispectral imaging.

The implementation of this method was integrated into the Gerbil multispectral analysis framework [10] and will be released as free software on http: //gerbil.sf.net.

\section{References}

[1] J. Canny, "A Computational Approach to Edge Detection," IEEE Transactions on Pattern Analysis and Machine Intelligence, vol. 8, no. 6, pp. 679-698, 1986.

[2] A. Cumani, "Edge detection in multispectral images," CVGIP: Graphical Models and Image Processing, vol. 53, no. 1, pp. 40-51, 1991.

[3] P.E. Trahanias and A.N. Venetsanopoulos, "Color edge detection using vector order statistics," IEEE Transactions on Image Processing, vol. 2, no. 2, pp. 259-264, 1993.

[4] C. Drewniok, "Multi-spectral edge detection. Some experiments on data from Landsat-TM," International Journal of Remote Sensing, vol. 15, no. 18, pp. 3743-3765, 1994.

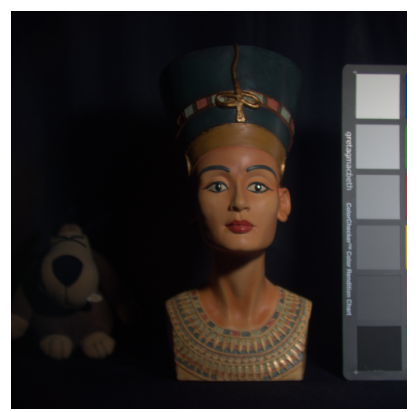

(a) Original image in sRGB

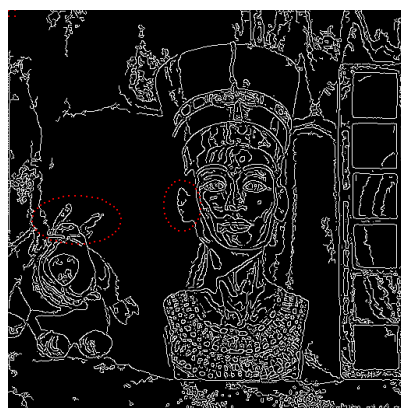

(c) Orig. method, 16x16 SOM; A

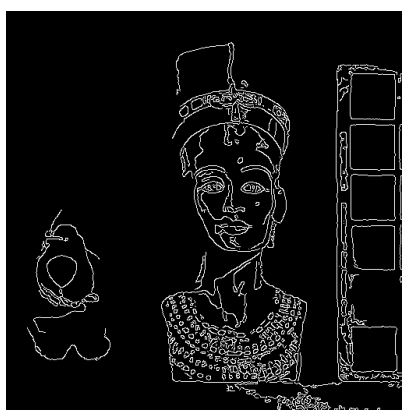

(e) Orig. method, 16x16 SOM; B

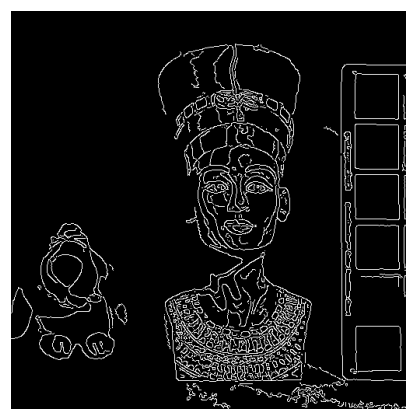

(b) $256 \times 1 \mathrm{SOM}$

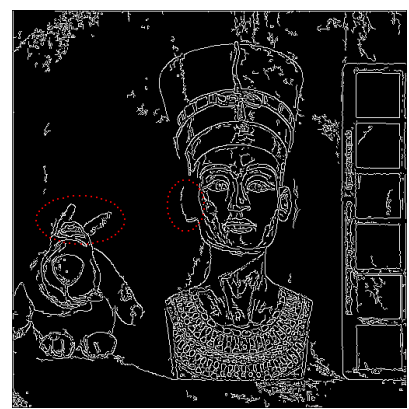

(d) Our method, 16x16 SOM; A

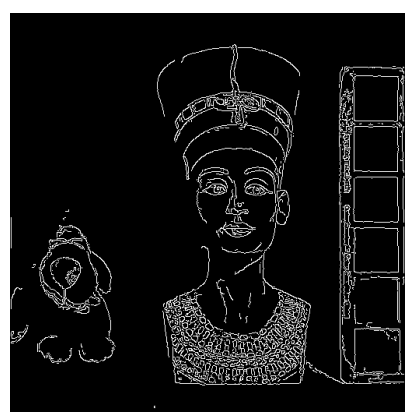

(f) Our method, 16x16 SOM; B
Fig. 5. Edge detection results. Canny parameter criteria: A) best object contour preservation; B) minimum fine-grained noise introduced by object/background texture. Marked in red are object parts that are not visible in the sRGB representation.

[5] P.J. Toivanen, J. Ansamäki, J.P.S. Parkkinen, and J. Mielikäinen, "Edge detection in multispectral images using the selforganizing map," Pattern Recognition Letters, vol. 24, no. 16, pp. 2987-2994, 2003.

[6] T. Kohonen, Self-organizing maps, vol. 30 of Springer series in information sciences, Springer, 3rd edition, 2001.

[7] F. Yasuma, T. Mitsunaga, D. Iso, and S. K. Nayar, "Generalized Assorted Pixel Camera: Post-Capture Control of Resolution, Dynamic Range and Spectrum," IEEE Transactions on Image Processing, vol. 99, Mar 2010.

[8] H. Sagan, Space-filling curves, Universitext. Springer, 1994.

[9] L. Biehl and D. Landgrebe, "Documentation for multispec," https://engineering.purdue.edu/ biehl/ MultiSpec/documentation .html, Jan 2011.

[10] J. Jordan and E. Angelopoulou, "Gerbil - A Novel Software Framework for Visualization and Analysis in the Multispectral Domain," VMV 2010: Vision, Modeling and Visualization, pp. 259-266, 2010. 